\title{
Analyse des sauts et de la course à pied par accélérométrie
}

\author{
Guillaume Laffaye $^{1}$, Boris Jidovsteff ${ }^{2}$ \\ 1. UR CIAMS - Motor Control and Perception Group, Sport Sciences Department, \\ Bât. 335, Université Paris-Sud, 91405 Orsay, France \\ guillaume.laffaye@u-psud.fr \\ 2. Training and assessment of physical fitness, Department of Sport and \\ Rehabilitation Sciences, University of Liege \\ Allée des sports 4, 400 Liège, Belgique \\ b.jidovtseff@ulg.ac.be
}

\begin{abstract}
RÉSUMÉ. L'objectif de cette seconde partie de l'article est de faire le tour des possibilités et des limites qu'offre l'accéléromètrie dans la performance sportive. Plus spécifiquement, les accéléromètres peuvent être utilisés dans des tests de sauts (saut squat, saut avec contremouvement, saut sur place) afin de mesurer la détente, mais également d'autres variables mécaniques, comme la raideur mécanique, la force et la puissance. Lors des activités de course, ils peuvent être un outil intéressant pour mesurer la vitesse de déplacement, la fréquence et un ensemble de paramètres utiles pour un entraîneur ou pour la compréhension même des phénomènes lors de ce mode de locomotion.

ABSTRACT. The goal of this chapter is to understand the interests and limits of the accelerometry for assessing the performance in sport. More specifically, accelerometers are used during jumping tests (squat jump, countermovement jump, hopping on place test) to measure jump height, power, force and leg stiffness as well. During running, they are used for assessing velocity, step frequency, and other useful parameters for trainers or scientists in the understanding of this mode of locomotion.

MOTS-CLÉS : saut, validation, reproductibilité, précision, mesure, raideur mécanique, force, puissance.

KEYWORDS: jump, validity, repeatability, accuracy, leg stiffness, force, power.
\end{abstract}

DOI:10.3199/I2M.14.3-4.229-249 (C) 2014 Lavoisier 


\section{L’accélérométrie et les sauts}

\subsection{La mesure de la détente et autres variables associées}

La mesure de la détente intéresse de nombreux pratiquants, entraîneurs et scientifiques. En effet, un protocole rigoureux permet non seulement de connaitre la performance lors d'une épreuve spécifique de sauts, mais également un ensemble de variables associées comme l'impulsion verticale développée, la puissance mécanique externe produite, une estimation d'une valeur que certains auteurs définissent comme "une raideur mécanique » globale des membres inférieurs ou encore le temps de contact lors de sauts enchaînés. Pour voir si un entraînement améliore la détente ou comparer la performance entre plusieurs sportifs, il est nécessaire d'avoir une précision dans la mesure de ces variables. On peut définir la détente verticale comme l'élévation du centre de masse du sauteur, différence entre l'altitude maximale atteinte dans la phase aérienne du saut et l'altitude à l'instant de décollage.

Les tests de détente classiques sans instrumentation consistent souvent à déduire la position de la main sur une toise après un saut de celle mesurée en position debout la main le long de la toise. Mais de nombreux biais jalonnent ce test désormais classique, créé aux États-Unis en 1921 sous le nom de «Sargent Test »(Sargent 1921) qui servait à tester la condition des jeunes militaires. Il consistait à réaliser un saut vertical avec contre-mouvement.

De nombreux scientifiques se sont par la suite attachés à déduire de ce test la puissance mécanique produite par les membres inférieurs à travers une équation de régression simple. La plus célèbre est celle de Lewis (Fox et Mathews, 1974) qui permet de connaitre sa puissance moyenne lors du saut selon la formule suivante ( $m$ pour la masse du sujet et $H$ pour la hauteur atteinte lors du saut):

$$
\text { Pmoy }(\text { Watt })=\sqrt{4.9} \cdot M(\mathrm{~kg}) \cdot \sqrt{H}(\mathrm{~m})
$$

Le pic de puissance ou puissance maximale, quant à lui, est estimé à partir de la formule de Sayers (Sayers et al., 1999):

$$
\operatorname{Pmax}(W a t t)=60.7 \times H(\mathrm{~cm})+45.3 \times M(\mathrm{~kg})-2055
$$

D'autres formules dérivées ont tenté de gagner en précision par la suite, comme celle de Harman (Harman et al,. 1991) ou encore de Johnson et Bahamonde (1996). Cependant l'ensemble de ces approches repose sur des erreurs théoriques fondamentales auxquelles s'ajoutent des approximations expérimentales non négligeables :

D'un point de vue fondamental, la mesure de l'élévation de la main n'est pas représentative de celle du centre de masse global du corps. Le corps humain étant un système déformable donc non rigide, le vecteur qui relie son centre de masse au 
centre de gravité de la main n'est pas constant. Par ailleurs, les formules (1) et (2) calculent une puissance au cours de la phase aérienne en ramenant ce calcul à celui de la variation de l'énergie potentielle du centre de masse dans la phase ascendante $\mathrm{du}$ saut, après le décollage du sujet. Or c'est pendant la phase d'impulsion qui précède qu'il convient d'évaluer la puissance mécanique produite par les muscles actionneurs du mouvement. Une fois décollé, le sauteur suit une loi balistique qui dépend uniquement de la valeur de la vitesse du centre de masse acquise à l'instant du décollage.

Par ailleurs, des biais expérimentaux peuvent être soulevés :

- d'une part, le sauteur peut jouer sur la position de son épaule lors de l'acmé de son saut pour gagner quelques centimètres par rapport à la position de référence debout et ainsi être surévalué ;

- d'autre part, la mesure entre la position debout et la position réelle lors du décollage est fondamentalement différente, car au moment du décollage le sauteur a ses pieds en extension complète et non pas uniquement collés au sol. Cela signifie que selon la taille du pied mais également les possibilités d'extension plantaire maximale de la cheville, la différence entre deux sauteurs avec la même détente peut être importante et uniquement liée à des aspects anthropométriques et non neuromusculaires.

En résumé, ce type de test et l'analyse des résultats ne repose pas sur un raisonnement scientifique correct. Ni la détente verticale mesurée, ni la puissance calculée lors de ces tests ne sont correctes. Ces données ne sont pas représentatives de la performance réalisée par le sauteur.

Une alternative est proposée par Samozino et collaborateurs qui ont proposé en 2008, une méthode d'estimation de la puissance mécanique externe $\mathrm{P}$, de la force verticale $\mathrm{F}$, action du sujet sur le sol et de la vitesse verticale du centre de masse lors d'un saut squat (Samozino et al., 2008).

La force verticale est calculée selon l'équation suivante :

$$
\mathrm{F}=\mathrm{m} \times 9,81 \times\left(\mathrm{h} / \mathrm{h}_{\mathrm{PO}}+1\right)
$$

La puissance $(\mathrm{W})$, quant à elle s'obtient ainsi :

$$
P=m \times 9,81 \times\left(\frac{h}{h p o}+1\right) \sqrt{9,81 \times h / 2}
$$

Avec m, la masse du sujet; $h_{P O}$ est la distance pendant laquelle le sauteur applique une force supérieure à son poids. Elle est mesurée en faisant la différence de la hauteur de sa jambe au grand trochanter (centre de rotation de la jambe) en position semi fléchi $\left(90^{\circ}\right.$ d'angulation de genou) et la position d'extension maximale mesurée préalablement en position couché, pied en extension plantaire maximale, 
tout cela en lecture directe sur une toise. La hauteur $\mathrm{h}$ du saut est mesurée par l'équation (6).

Selon les auteurs, cette approche démontre une différence de moins de $3 \%$ avec la mesure effectuée avec une plateforme de force. Il faut préciser que cette méthodologie est soumise aux mêmes hypothèses et limites rencontrées lors de l'utilisation de l'OptoJump ou du tapis de Bosco qui «estime» la hauteur sautée à partir de la mesure du temps de vol. Une autre alternative à ces tests dans des conditions écologiques sans avoir recours aux plateformes de force, est l'utilisation d'accéléromètres. Les formules se basent sur les lois de la balistique pour mesurer l'élévation du centre de masse. Les deux méthodes utilisées sont basées soit sur le temps que l'athlète reste en l'air (méthode du temps d'envol), soit sur la vitesse de décollage. La hauteur atteinte $H$ lors du saut se mesurera donc selon la vitesse d'envol :

$$
H=\frac{V d e c^{2}}{2 g} \text { en } \mathrm{m}
$$

avec Vdec la vitesse verticale de décollage et g la gravité $\left(9,81 \mathrm{~m} \cdot \mathrm{s}^{-2}\right)$, ou selon la formule du temps d'envol :

$$
H=\frac{1}{8} g T^{2} \text { en } \mathrm{m}
$$

avec g la gravité $\left(9,81 \mathrm{~m} \cdot \mathrm{s}^{-2}\right)$ et $T$ le temps d'envol. Cette formule repose sur l'hypothèse que dans la phase aérienne, la parabole du centre de masse est symétrique ce qui revient à considérer que les positions verticales du centre de masse évaluées par rapport au sol sont égales. Or cette condition n'est que très rarement vérifiée.

Par ailleurs, l'appareil mesure l'accélération et une routine de calcul généralement intégrée à l'appareil calcule la vitesse en intégrant les données accélérométriques selon la formule :

$$
\mathrm{v}_{\mathrm{V}}(\mathrm{t})=\int \mathrm{a}_{\mathrm{V}} \mathrm{dt}+\mathrm{v}_{\mathrm{V}}\left(\mathrm{t}_{0}\right) \text { en } \mathrm{m} \cdot \mathrm{s}^{-1}
$$

avec $V_{v}$ la vitesse verticale, $V_{v}\left(t_{0}\right)$ la vitesse à l'instant initial, $a_{v}$ l'accélération verticale. Le déplacement $Z$ du capteur sera, quant à lui, déduit de l'accélération en intégrant deux fois les données accélérométriques :

$$
Z(t)=\iint a_{v} d t+Z\left(t_{0}\right) \text { en } m
$$

$\mathrm{Z}\left(\mathrm{t}_{0}\right)$ est la position initiale du centre de masse.

La force verticale de réaction du sol ainsi que la puissance mécanique externe suivant l'axe vertical sont, quant à elles, déduites de la deuxième loi de Newton :

$$
F_{v}=m\left(a_{v}+g\right) \text { en N.kg-1 }
$$




$$
P_{v}=F_{v} \cdot v_{v} \text { en } \mathrm{W} \cdot \mathrm{kg}^{-1}
$$

Les formulations (7), (8), (9) et (10) sont correctes si et seulement si $a_{v}(t)$ représente bien l'accélération verticale du centre de masse du sauteur condition non respectée, puisque par définition le centre de masse est un point virtuel dont la position varie suivant la déformation du corps. L'accéléromètre ne peut donc pas évaluer $\mathrm{a}_{\mathrm{v}}(\mathrm{t})$. De plus, le capteur mesure dans son propre référentiel dont l'orientation varie au cours du temps.

On distingue quatre grands types de saut pour mesurer la détente ou des qualités neuromusculaires (figure 1):

- le saut squat, qui consiste à sauter à partir d'une position semi-fléchie $\left(90^{\circ}\right)$ sans réaliser de contre-mouvement vers le bas. Ce test mesure essentiellement la capacité de l'athlète à développer une force concentrique des extenseurs de la cuisse dans un laps de temps très court. Il est ainsi un bon indicateur de la force explosive.

- le saut avec contremouvement consiste à sauter à partir d'une position debout en réalisant un contremouvement vers le bas. Le contremouvement vers le bas permet d'étirer les tendons et d'emmagasiner de l'énergie élastique (van Ingen Schenau et al., 1997) en sollicitant le cycle étirement-renvoi qui permettra un gain notable de performance. Il est le test de référence lorsqu'on parle de détente verticale.

- le saut de contre-haut ou « drop jump » chez les anglophones consiste, à partir d'un contrehaut de hauteurs variables (classiquement entre 30 et $70 \mathrm{~cm}$ ), à se laisser tomber puis sauter sans délai dès la réception. La chute est utilisée pour pré-activer les muscles extenseurs et produire une force verticale plus grande au contact au sol qui selon certaines hypothèses permettraient d'utiliser l'élasticité musculotendineuse et le réflexe myotatique pour sauter plus haut (Laffaye et al., 2006). La mesure des qualités pliométriques peut se faire aisément en réalisant la batterie de ces 3 tests. Si la meilleure performance est obtenue lors du saut squat, l'athlète est explosif (définit ici comme la vitesse d'augmentation de la force musculaire) avec peu de «qualités pliométriques».Si au contraire sa performance maximale est obtenue lors du saut de contre-haut, il possède de « bonnes qualités pliométriques ». Notons que le terme qualité pliométrique est un terme de terrain.

- le saut sur place, ou saut de réactivité consiste à enchaîner des sauts sur place avec la double contrainte de sauter haut avec un temps d'impulsion au sol le plus court possible. On peut estimer alors une valeur pouvant être assimilée à une raideur mécanique globale des membres inférieurs... Ce modèle considère le corps humain comme une masse $m$ posée sur un ressort sans masse de longueur $L$, déformable et de raideur $k$. Cette approche macroscopique théorique peut offrir des indications sur la capacité du système musculo-tendineux à emmagasiner puis restituer l'énergie tel un ressort.

Dans l'ensemble de ces sauts, afin de limiter les biais et avoir la plus grande précision possible, les protocoles accélérométriques imposent de sauter avec les 
mains bloqués sur la taille. L'appareil est alors fixé sur une ceinture au niveau des hanches.

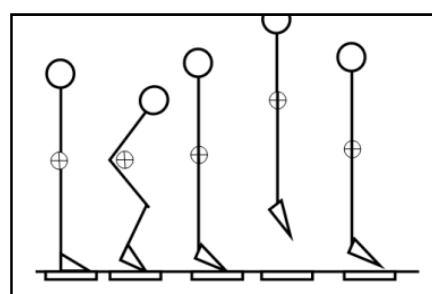

Saut avec contremouvement

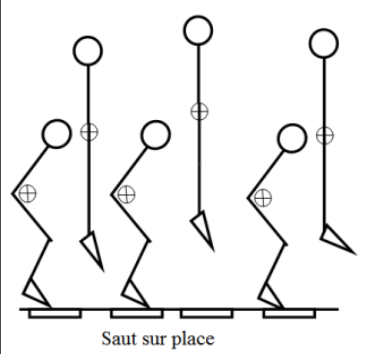

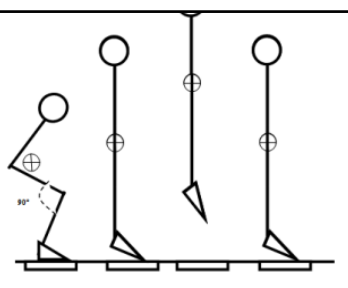

Saut squat

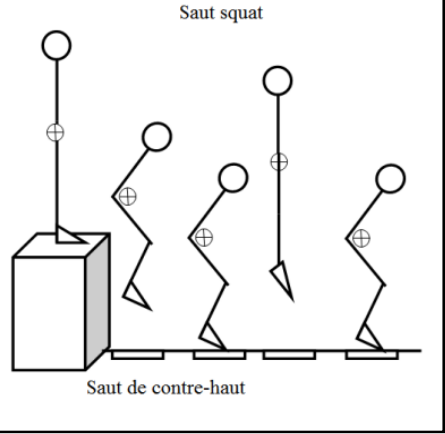

Figure 1. Illustration en stick dequatre types de sauts.

\subsection{Le saut squat}

\subsubsection{La mesure de la performance verticale}

Dans une étude récente, une équipe de chercheurs suisses (Casartelli et al., 2010) a testé la fiabilité et la reproductibilité de la performance en saut en utilisant un accéléromètre (Myotest SA) attaché à une ceinture lors de saut squat. Cette valeur de reproductibilité dépend à la fois de la reproductibilité de l'outil ainsi que celle de l'athlète à reproduire une même performance. On entend par validité, la capacité de l'outil à produire une mesure proche de la valeur vraie (valeur étalon ou valeur obtenue à partir d'un autre outil considéré comme référence). Casartelli et al., 2010 ont choisi de comparer les valeurs produitent par, le Myotest avec celle obtenue par un optojump. Les résultats ne permettront qu'une comparaison des 2 systèmes mais en aucun de déterminer si l'un est plus valide que l'autre. Précédemment les valeurs de temps de vol de l'optojump ont été comparées avec celles provenant d'une plateforme de force (Glatthorn et al., 2011). L'optojump consiste en deux barres parallèles posées au sol et munies de cellules photoélectriques qui enregistrent le temps d'envol et le temps de contact a sol (avec une précision au $1 / 1000^{\mathrm{e}}$ de seconde) afin notamment de déduire la hauteur du saut selon l'équation (6). Les mesures accélérométriques sont étudiées selon les deux méthodes : celle du temps d'envol et celle de la vitesse de décollage. La fréquence d'échantillonnage du 
Myotest est réglée à $500 \mathrm{~Hz}$ (500 données par seconde). Les équations calculées par les trois appareils sont celles présentées précédemment (Casartelli et al., 2010) : la méthode de calcul de la hauteur sautée basée sur la vitesse de décollage est représentée par myotest-V et la méthode de calcul de la hauteur sautée du temps d'envol par Myotest-T.

Comme le montre la figure 2, les deux myotests surestiment les performances de manière significative par rapport à l'appareil de référence (optojump). Le biais systématique est de $+6,99 \mathrm{~cm}$ pour le myotest- $T$ et de $+5,66 \mathrm{~cm}$ pour le myotest- $\mathrm{V}$. On entend par biais systématique la tendance générale d'une mesure d'être différente de la réalité (ici de l'optojump) selon une direction particulière (négative ou positive). On mesure également l'erreur aléatoire qui rend compte du degré auquel la mesure répétée varie pour un individu donné (Atkinson et Nevill, 1998). Cette erreur aléatoire est de $\pm 2,54 \mathrm{~cm}$ pour myotest- $T$ contre $\pm 12,64 \mathrm{~cm}$ pour myotest-V. La différence est donc importante par rapport à la mesure de référence. Quant à la reproductibilité, elle est importante pour myotest-T (ICC entre 0,92 et 0,96 ) et acceptable pour le myotest-V (entre 0,70 et 0,90). En d'autres termes, les auteurs expliquent que le Myotest-T même s'il surévalue la performance montre une bonne validité et reproductibilité, ce qui n'est pas le cas du myotest-V.

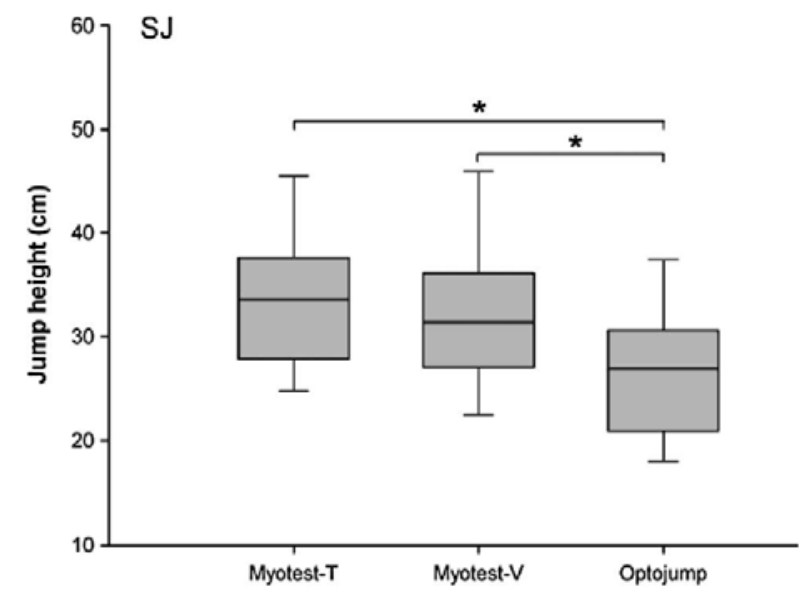

Figure 2. Hauteur comparative de saut squat obtenue par accélérométrie selon la méthode du temps d'envol (Myotest-T), de la vitesse d'envol (Myotest-V) et avec un optojump (d'après Casartelli et al,. 2010). * représente une différence significative avec l'optojump à p<0,05: COPYRIGHT JSCR

Une autre étude (Choukou et al., 2014) compare la performance en saut squat (méthode temps d'envol) par accélérométrie avec celle obtenue par plateforme de force $(1000 \mathrm{~Hz})$. Les résultats montrent le même biais systématique $(+5,6 \mathrm{~cm})$ et 
une erreur aléatoire élevée $( \pm 11,7 \mathrm{~cm})$ avec une différence statistiquement significative. La reproductibilité est également élevée (corrélations intra-classe comprises entre 0,82 et 0,84 ).

Les auteurs précisent que la surestimation du temps de vol provient de la méthodologie utilisée par l'accéléromètre pour mesurer le temps d'envol. Théoriquement, le temps d'envol correspond au temps entre lequel le sauteur quitte le sol (décollage) et atterrit. Le Myotest estime le temps d'envol entre la différence de temps entre le pic positif de vitesse verticale (phase propulsive du saut) et le pic négatif de vitesse verticale (atterrissage). Cependant, la vitesse positive maximale se produit légèrement avant le décollage et le pic de vitesse négative légèrement après l'atterrissage, surévaluant ainsi la valeur réelle (Choukou et al,. 2014 ; Casartelli et al., 2010)

\subsubsection{Précision de la mesure de la force et de la puissance}

Comme nous avons pu le voir précédemment, si l'accélération du centre de masse est correctement mesurée, les lois newtoniennes permettent d'avoir directement accès à la force à partir de l'accélération. Une étude récente (Choukou et al., 2014) montre que la force relative (force divisée par le poids du sujet) n'est pas significativement différente entre les valeurs obtenues par accéléromètre et plateforme de force. Le biais systématique est de $-1,4 \mathrm{~N} \cdot \mathrm{kg}^{-1}$ et l'erreur aléatoire de $\pm 2,4 \mathrm{~N} \cdot \mathrm{kg}^{-1}$. Il faut noter que cette erreur aléatoire entrainera également des écarts importants pour la détermination de la vitesse et du déplacement du centre de masse. Les ICC lors de test/retest sont comprises entre 0,85 et 0,92 ).

La puissance mécanique externe, quant à elle, est calculée en faisant le produit scalaire de la force de réaction du sol par la vitesse du centre de masse. Si les valeurs obtenues par accélérométrie sont reproductibles, avec des corrélations intra-classes comprises entre 0,74 et 0,83 , la validité n'est pas bonne, avec une différence significative avec la valeur obtenue par la plateforme de force. En effet, le biais systématique est de $+11,7 \mathrm{~W} \cdot \mathrm{kg}^{-1}$, l'erreur aléatoire est de $\pm 16,9 \mathrm{~W} \cdot \mathrm{kg}^{-1}$, et les corrélations intra-classes comprises entre 0,18 et 0,31 , ce qui extrêmement faible. On admet qu'une valeur $>0,90$ est une valeur excellente, $>0,80$ une valeur bonne (Bland et Altman, 1990). L'explication de valeur erronée lors de la mesure de la puissance s'explique par une dérive de la mesure de la vitesse, qui est surestimée par rapport aux valeurs mesurées par la plateforme de force. En outre, l'analyse de la vitesse par la méthode de Bland-Altman montre une hétéroscédasticité des valeurs, c'est-à-dire que l'écart entre les deux valeurs augmente au fur et à mesure qu'augmente les performances. Par conséquent, le produit scalaire de la force par la vitesse pour calculer la puissance mécanique externe augmentera donc cette erreur rendant l'évaluation de la puissance par accélérométrie trop loin de la réalité pour pouvoir être prise en compte.

Une autre étude dans laquelle le myotest a été attaché à un bâton (Bampouras et al., 2013) montre une corrélation élevée entre une plateforme de force et le myotest 
pour la force $(r=0,82)$ même si la différence mesurée du biais est significative. Les auteurs expliquent cette différence comme étant due au fait que l'accélération mesurée sur la barre est différente de celle de l'athlète, ce qui surestime la force produite. Cela se retranscrit sur le pic de force maximum, qui se produit juste avant la descente maximale du sujet sur la plateforme de force, alors que l'accéléromètre fixé sur la barre en fin de séquence proximo-distale enregistre l'accélération maximale au point distal à laquelle l'accélération linéaire de l'ensemble du système se rajoute. Au final, le pic d'accélération de l'appareil s'en retrouve décalé après le pic de force réel. La reproductibilité est haute pour la force (ICC $=0,90$, $\mathrm{CV}=2,1 \%$ ), la vitesse ( $\mathrm{ICC}=0,84, \mathrm{CV}=3,2 \%$ ) et la puissance ( $\mathrm{ICC}=0,80$, $\mathrm{CV}=3,3 \%)$.

Enfin, lors de squats avec des charges sur les épaules (de $20 \mathrm{~kg}$ à $80 \mathrm{~kg}$ ), les valeurs obtenues par accélérométrie (Crewther et al., 2010) sont modérément à fortement corrélées avec celles d'une plateforme de force, entre $r=0,87-0,97$ en ce qui concerne le pic de force et entre $r=0,66$ et $r=0,90$ pour le pic de puissance. Un biais systématique significatif ( $>170 \mathrm{~N}$ pour la force) est observé uniquement dans la condition $60 \mathrm{~kg}$ en ce qui concerne la force, le biais ainsi que l'erreur systématique diminuant avec la charge (force et puissance). La différence est expliquée pour les mêmes raisons que dans l'étude précédente à cause de l'éloignement de l'appareil du centre de masse.

\subsection{Le saut avec contremouvement}

\subsubsection{Précision de la mesure de la performance verticale}

De nombreuses études se sont intéressées à la mesure de la performance lors du saut avec contremouvement (Bubanj et al., 2010 ; Casartelli et al., 2010 ; Choukou et al., 2014; Castagna et al., 2013 ; McMaster et al., 2013 ; Haff et al., 2011 ; Nuzzo et al., 2011), montrant par là même qu'il s'agit du standard des tests de détente.

Comparativement à un optojump, la performance en CMJ (Casartelli et al., 2010) montre une faible validité du myotest par la méthode de la vitesse de décollage, avec notamment une forte hétéroscédasticité, c'est-à-dire que l'erreur augmente avec l'augmentation des performances. La méthode de mesure par temps d'envol est quant à elle correcte, malgré une surestimation significative de la performance, avec un biais de $+7,24 \mathrm{~cm}$ et une erreur aléatoire de $\pm 2,82 \mathrm{~cm}$. La performance est hautement reproductible $(\mathrm{ICC}=0,96)$.

Comparé à des résultats de plateforme de force (Choukou et al., 2014), la performance est légèrement surévaluée, le biais étant de $+3,6 \mathrm{~cm}$. Malgré ce biais, les valeurs sont hautement reproductibles ( $\mathrm{ICC}>0,80, \mathrm{CV}=4,31 \%)$. Une telle reproductibilité $(\mathrm{ICC}=0,88)$ est notée dans une autre étude sur le temps d'envol (Castagna et al., 2013). La corrélation dans cette étude est de $r=0,89$ avec la plateforme de force. Le temps d'envol mesuré par le myotest est en moyenne 6,4 \% plus long que par la plateforme de force, ce qui explique la surévaluation notée dans 
toutes les études. L'étude de Nuzzo (Nuzzo et al., 2011) montre également la grande reproductibilité des performances ( $\mathrm{ICC}=0,95, \quad \mathrm{CV}=3,3 \%)$ avec une hétéroscédasticité comme dans l'étude de Casartelli.

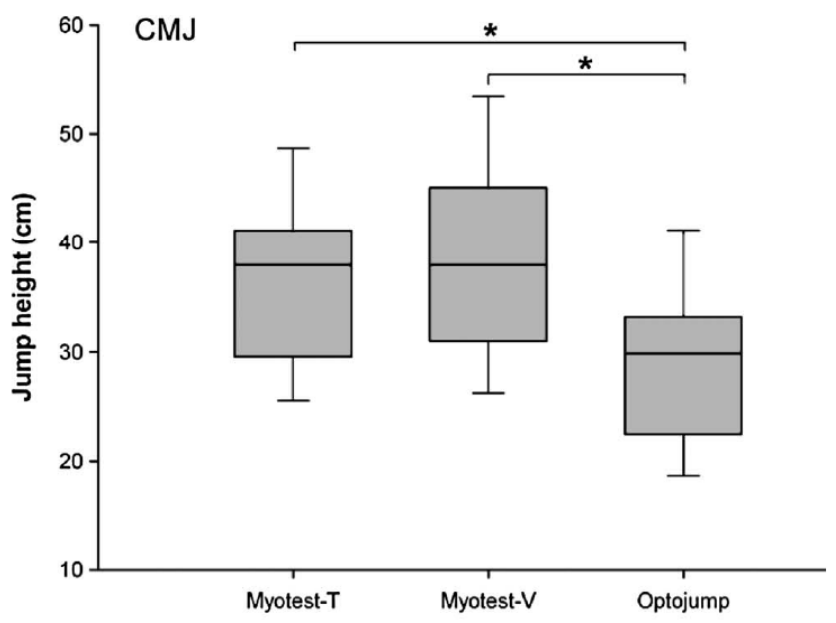

Figure 3. Hauteurs comparatives de saut avec contremouvement obtenues par accélérométrie selon la méthode du temps d'envol (Myotest-T), de la vitesse d'envol (Myotest-V) et avec un optojump (D'après Casartelli et al., 2010). * représente une différence significative avec l'optojump à p<0,05: COPYRIGHT JSCR

Enfin, deux études montrent l'importance du placement de l'accéléromètre et de la fréquence d'acquisition sur la fiabilité de la mesure. Dans une première étude (McMaster et al., 2013) l'accéléromètre est placé soit sur le côté de la hanche, au niveau de la tête du fémur, soit sur un bâton positionné sur les épaules. Le pic de vitesse est étudié comme variable afin de mesurer la performance. Les valeurs sont assez similaires à celle de la PF ( $2 \%$ d'écart) en ce qui concerne l'accéléromètre à la barre, mais dérive de manière trop importante lorsqu'il est placé à la hanche (21\% d'écart). Ces résultats sont probablement dus à un placement de l'accéléromètre sur un axe de rotation (grand trochanter) mais également à une fréquence d'acquisition insuffisante $(200 \mathrm{~Hz})$. Ceci focalise sur l'importance de ces deux variables.

En effet, seule une étude a testé l'influence de la fréquence d'acquisition (Haff et al., 2011) sur l'ensemble des variables classiquement étudiées lors de sauts. Les résultats montrent une haute reproductibilité à $500 \mathrm{~Hz}$ (ICC entre 0,87 et 0,94$)$ puis une baisse de cette reproductibilité proportionnellement à la fréquence d'acquisition. Les auteurs concluent qu'en dessous de $250 \mathrm{~Hz}$, les valeurs sont modérément voire peu reproductibles. 
En conclusion, toutes ces études montrent une haute reproductibilité des performances verticales en saut avec contremouvement à partir du moment où la fréquence est supérieure à $250 \mathrm{~Hz}$ et l'appareil placé de manière à éviter des rotations. Enfin, le biais systématique qui varie entre $3,6 \mathrm{~cm}$ et $7,2 \mathrm{~cm}$ selon les études est dû à une surestimation du temps d'envol, qui ne change rien à la fiabilité de la mesure, mais qui devra être considérée si l'on souhaite comparer les résultats avec ceux obtenus par d'autres systèmes de mesure. Finalement, ce qui sera le plus important est de mesurer la détente verticale à partir du temps d'envol et non pas de la vitesse qui elle, est trop aléatoire.

\subsection{Le saut sur place}

Le saut sur place que l'on appelle également sauts répétés ou sauts rebonds a été développé afin de mesurer la raideur mécanique $(k)$ ou encore l'indice de réactivité (IR). Ces indices s'appuient sur le paradigme du système masse ressort, qui considère que dans les activités nécessitant un cycle étirement-renvoi, comme c'est le cas dans le sprint ou le saut sur place, le système musculo-tendineux se comporte comme un ressort capable d'emmagasiner de l'énergie dans la phase excentrique et de la restituer dans la phase concentrique. On peut dès lors mesurer la raideur mécanique globale des membres inférieurs de ce ressort $\left(\mathrm{en} \mathrm{kN} \cdot \mathrm{m}^{-1}\right)$, classiquement en faisant le rapport de la force maximale concentrique $\left(\mathrm{F}_{\mathrm{c}}\right)$ obtenue sur l'abaissement du centre de masse $\left(\Delta_{\mathrm{COM}}\right)$, c'est-à-dire l'abaissement du centre de gravité pendant le saut.

$$
k_{\text {leg }}=F_{c} / \Delta_{C o M}\left(\mathrm{kN} \cdot \mathrm{m}^{-1}\right)
$$

On mesurera également l'indice de réactivité (IR) comme le rapport du temps d'envol (FT) sur le temps de contact au sol (CT) :

$$
\mathrm{IR}=\mathrm{FT} / \mathrm{CT} \text { (sans unité) }
$$

Avant de mesurer la raideur mécanique, il est nécessaire de vérifier que le comportement du sujet est bien un comportement de ressort, c'est-à-dire qu'il présente une linéarité entre la force et la compression (figure 4). Par convention, on admet qu'une linéarité respectée avec un $\mathrm{r}^{2}>0,80$ entre dans le modèle masse-ressort.

Le protocole de sauts répétés consiste à enchaîner une série de sauts (classiquement entre 5 et 10) avec la double contrainte de sauter haut malgré un temps d'impulsion court au sol. Le but est alors d'obtenir la plus grande valeur de raideur. L'indice de réactivité (IR) est une variable dérivée, qui permet notamment d'éviter des biais de certains comportements qui tendent à surévaluer artificiellement les valeurs de raideur. En effet, un sujet qui chercherait à avoir un temps extrêmement court sans pour autant sauter haut (il appuie juste la plante du pied et le relève rapidement) conduirait à des valeurs de raideur incongrues en prenant en 
compte une valeur de choc au sol (ou pic passif de la force) comme valeur de force concentrique maximale.

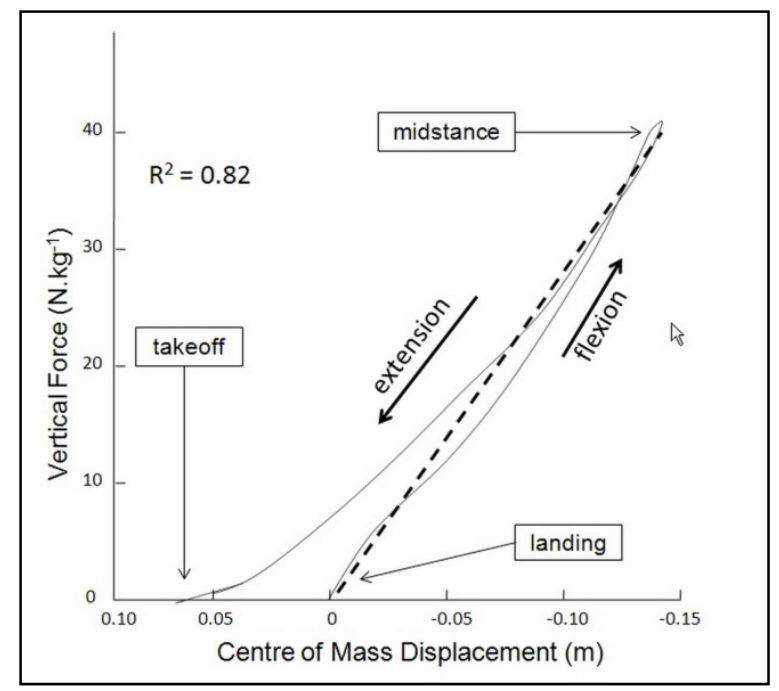

Figure 4. illustration de la linéarité force-compression dans un saut sur place d'après le modèles masse-ressort (D'après Choukou et al., 2014)

Deux études, l'une française, l'autre suisse, ont testé la possibilité d'enregistrer la raideur mécanique, l'IR, le temps de contact et la hauteur du saut lors de sauts sur place (Choukou et al., 2014 ; Casartelli et al., 2010).

L'étude de l'équipe suisse (Casartelli et al., 2010) s'est focalisé sur la hauteur des sauts en comparaison avec l'optojump, et montre une surestimation du Myotest$\mathrm{T}$ de $+7,41 \mathrm{~cm}$ en biais systématique (contre $+12,31 \mathrm{~cm}$ pour le myotest-V) et une erreur aléatoire de $\pm 2,72 \mathrm{~cm}$ (contre $\pm 16,91 \mathrm{~cm}$ pour le myotest- $\mathrm{V}$ ). Les différences de hauteur sont significatives, pour les mêmes raisons de détection de la vitesse maximale que lors des deux autres types de sauts. La reproductibilité en test/retest est meilleure pour le myotest-T (ICC entre 0,86 et 0,96 ) que pour le myotest-V (ICC entre 0,32 et 0,73). Choukou et al. (2014) ont été trouvés en comparant accéléromètre et plateforme de force par l'équipe française avec la méthode du temps d'envol. Ils montrent avec un ICC entre 0,74 et 0,85 , un biais systématique de $+1,8 \mathrm{~cm}$ et une erreur aléatoire $\pm 15,3 \mathrm{~cm}$ Cette valeur de l'erreur aléatoire semble incompatible avec la réalisation de mesure de scientifique. Quant aux autres variables, seule l'étude française les a comparées aux valeurs obtenues sur plateforme de force. La raideur mécanique globale des membres inférieurs ne montre pas de différence significative entre les deux appareils. Toutefois le biais entre les 2 systèmes est de $+7,8 \mathrm{kN} \cdot \mathrm{m}^{-1}$ avec une erreur aléatoire de $\pm 12,7 \mathrm{kN} \cdot \mathrm{m}^{-1}$. 
L'ICC lors d'un test/retest est compris entre 0,86 et 0,92 avec un coefficient de variation de $6 \%$. La mesure du temps de contact est quant à elle significativement sous-évaluée par l'accéléromètre (biais : $-69 \mathrm{~ms}$; erreur aléatoire : $\pm 21 \mathrm{~ms}$ ). Cette sous-estimation systématique du temps de contact est due à la méthodologie employée pour le mesurer. En effet, l'appareil considère le temps de contact effectif, c'est-à-dire le laps de temps pendant lequel la force produite est supérieure au poids du sujet $(\mathrm{F}=\mathrm{m} \times \mathrm{g})$, ce qui n'est évidemment pas le cas de la plateforme de force qui mesure le temps de contact comme une valeur supérieure à zéro (figure 5). L'ICC est compris entre 0,88 et 0,93 avec un coefficient de variation de $6 \%$. Les valeurs de 1"'indice de réactivité obtenues sur plate forme de force et par le le Myotest ne sont pas différentes statistiquement (biais systématique de +0.4 et une erreur aléatoire de $\pm 0,9$ ). L'ICC est compris entre 0,94 et 0,96 et le coefficient de variation de $8 \%$. L'importance du CV devra être prise en compte dans l'interprétation des mesures de cet index. Il faut donc bien comprendre que l'outil le plus cohérent pour mesurer le temps de contact reste la plateforme de force. Un accéléromètre comme le Myotest fournira bien une valeur mais celle-ci ne correspondra pas au temps de contact effectif. La reproductibilité des mesures du Myotest permettra une comparaison entre différents test/retests, en revanche les différents calculs réalisés à partir de ses valeurs comporteront un biais de mesure. Connaissant ces limites, il conviendra de choisir l'outil le plus approprié en fonction des objectifs recherchés.

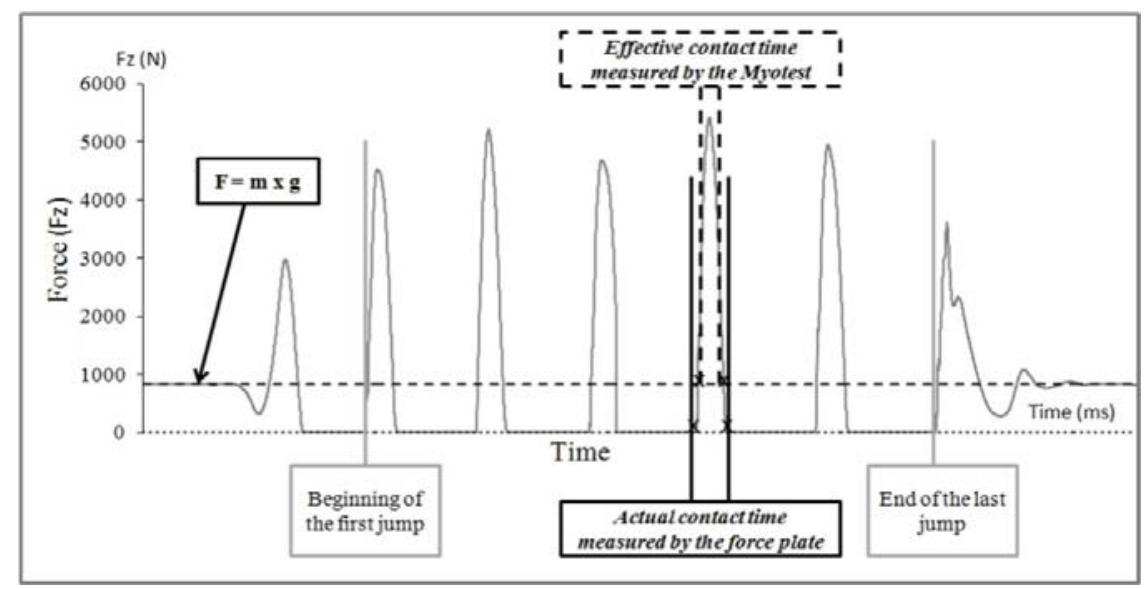

Figure 5. Comparaison entre le temps de contact réel mesuré par la plateforme de force et le temps de contact effectif mesurée par le Myotest

(d'après Choukou et al., 2014) 


\subsection{Les profils musculaires}

Plusieurs études montrent toutefois que lorsque l'on modifie quelque peu les consignes de réalisation des sauts, les paramètres biomécaniques se modifient. Les contextes de sauts ne sont pas les mêmes dans tous les sports et à l'intérieur d'un même sport, on peut également retrouver des impulsions avec plus ou moins de flexion de genou par exemple. Afin d'offrir un profil plus complet, il a été récemment proposé de réaliser avec un Myotest des séries de sauts sur place. Cinq modalités de saut différentes étaient proposées, allant de la recherche du temps de contact le plus court possible à la recherche de la hauteur de saut la plus élevée (Jidovtseff et al., 2012a). L'évaluation des 4 paramètres (hauteur de saut, temps de contact au sol, index de réactivité et raideur) a permis d'objectiver les relations qui les unissent (figure 6)
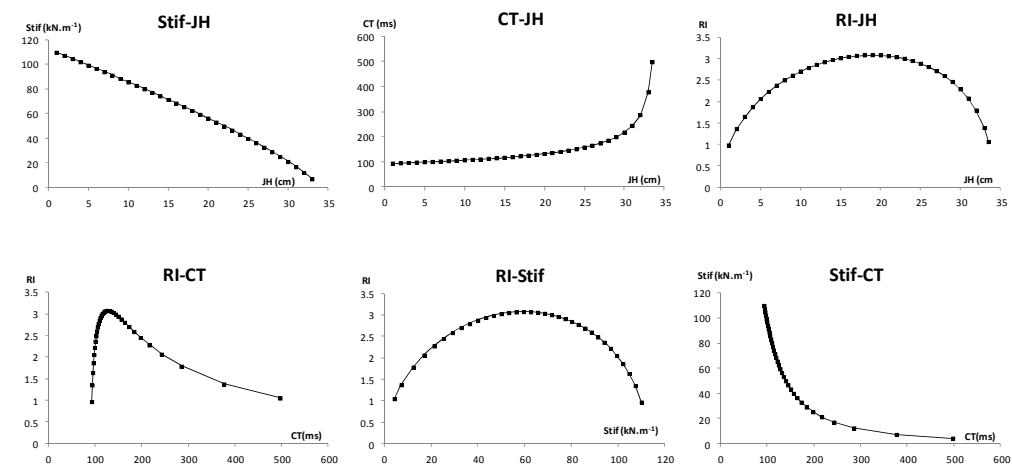

Figure 6. Relations établies entre la hauteur de saut (JH), le temps de contact (CT), l'index de réactivité (RI) et la raideur (Stif) établies lors d'un profil pliométrique après modélisation mathématique (Jidovtseff et al., 2012a)

Les résultats préliminaires (données personnelles, non publiées) montrent une répétabilité qui correspond aux normes des tests de saut. Cette nouvelle approche apparait prometteuse car elle offre en un protocole simple et progressif, une évaluation plus complète des qualités pliométriques. Des études doivent encore être réalisées afin de confirmer les résultats préliminaires et afin d'identifier les paramètres les plus pertinents.

\section{L’accélérométrie et la course}

Lors d'activités de course, la miniaturisation des accéléromètres permet leur utilisation en condition réelle de locomotion, Cet outil permet donc à moindre de 
cout de «sortir» du laboratoire. Comme toujours, il faudra analyser les résultats obtenus en prenant en considération les différentes limites des mesures réalisées. Dans ce contexte, il est possible d'obtenir une évaluation de certains paramètres de la foulée comme sa longueur, sa fréquence ou la symétrie, et également la vitesse de déplacement du coureur. Enfin, certains travaux ont tenté de coupler l'accéléromètre avec un cardio-fréquencemètre pour évaluer la dépense énergétique. Cette dernière restera très indirecte et approximative étant donné le fait qu'à la base la fréquence cardiaque ne la mesure pas directement.

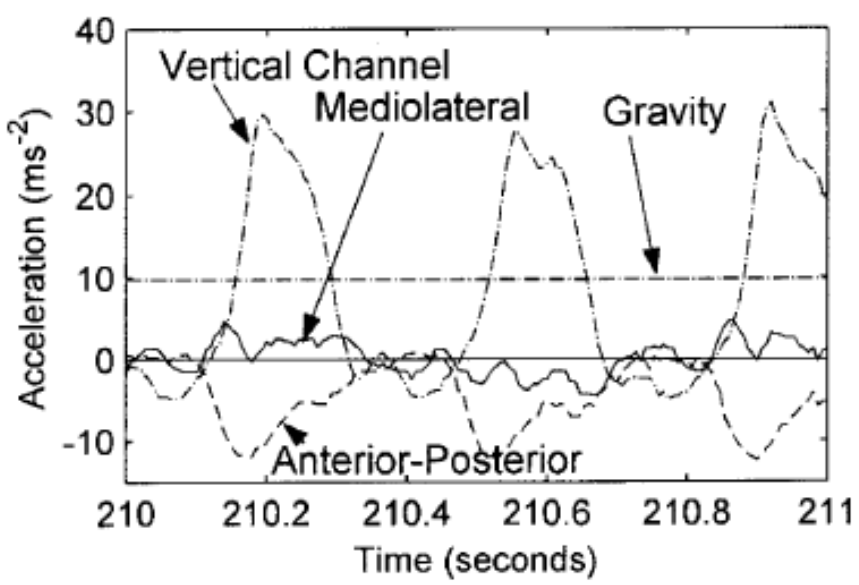

Figure 7. Signal accélérométrique typique obtenu selon 3 axes chez un athlète courant à $13 \mathrm{~km} / \mathrm{h}$. (D’après Wixted et al., 2007)

Une solution novatrice est proposée par la société Myotest, qui offre un appareil qui intègre l'ensemble des paramètres de courses (12 au total). Celui-ci, le Myotest run, permet notamment aux athlètes d'améliorer leur foulée sur la base de ses retours. Le tableau ci-dessous montre la répétabilité des variables mesurées lors de deux situations : à $10 \mathrm{~km} / \mathrm{h}$ et à $90 \%$ de la vitesse maximale aérobie des 32 athlètes testés (Jidovtseff et al., 2012b). Ces mesures montrent une bonne répétabilité des variables $(\mathrm{CV}<5 \%)$ dans les zones de vitesses testés, avec notamment une faible variation entre les valeurs obtenues sur tapis roulant et celle sur piste. Comme pour les autres tests réalisés par cet appareil, le temps de contact n'est pas le temps de contact réel, mais le temps que passe l'athlète lorsque la force qu'il développe est supérieure à son propre poids. Il faut préciser encore une fois que les valeurs de force présentées sont calculées en considérant par approximation que le Myotest mesure l'accélération du centre de masse, ce qui en réalité n'est pas le cas. Des études complémentaires devraient pouvoir préciser la validité de cet outil tout intégré. 
Tableau 1. Reproductibilité des paramètres de la foulée mesurée par Myotest Run sur 32 athlètes (d'après Jidovtseff et al., 2012b)

\begin{tabular}{ccc}
\hline Paramètres du Myotest Run & CV à $10 \mathrm{~km} / \mathrm{h}$ & $\begin{array}{c}\text { CV à } 90 \% \text { de } \\
\text { VMA }\end{array}$ \\
\hline Fréquence d'appui (nombre d'appui par mn) & 0,80 & 1,16 \\
Longueur de la foulée $(\mathrm{cm})$ & 0,86 & 1,17 \\
Temps de contact $(\mathrm{ms})$ & 1,79 & 2,42 \\
Distance parcourue pendant le temps de contact & 2,1 & 2,54 \\
(cm) & 1,99 & 1,50 \\
Angle de décollage du centre de gravité $\left(^{\circ}\right)$ & 0,35 & 0,65 \\
Angle de la jambe à l'atterrissage $\left(^{\circ}\right)$ & 2,11 & 2,21 \\
Mouvements verticaux du centre de gravité $(\mathrm{cm})$ & 2,77 & 4,16 \\
Raideur (kN/m) & 4,56 & 3,67 \\
Réactivité & 2,59 & 1,82 \\
Force moyenne à l'appui $(\mathrm{N} / \mathrm{Kg})$ & 1,9 & 1,6 \\
Énergie dépensée/km $(\mathrm{kcal})$ & 3,35 & 3,32 \\
\hline Index de régularité $(\%)$ & & \\
\hline
\end{tabular}

$C V=$ coefficients de variations, $V M A=$ vitesse maximale aérobie

\subsection{La vitesse de course}

La méthode classiquement utilisée consiste à évaluer la fréquence d'appuis à partir des chocs selon l'axe médiolatéral ou triaxial. Les à-coups sont calculés comme l'intégrale de l'accélération verticale au carré (selon un axe ou la somme des carrés des trois axes). La fréquence d'appui est calculée en général sur le nombre de fois que l'accélération verticale croise le zéro par unité de temps. La vitesse de course est approximée en partant du principe que la fréquence d'appui augmente de manière linéaire avec la vitesse. Ce principe n'étant pas toujours vérifié, car une variation de l'amplitude de la foulée peut également affecter la vitesse, il faudra rester prudent quant à l'interprétation de ces valeurs. La figure 8 présente un exemple de variation de fréquence (de 2,5 à $3,3 \mathrm{~Hz}$ ) lorsque la vitesse de course augmente de 9 à $21 \mathrm{~km} / \mathrm{h}$ (Wixted et al., 2005). Il semble que la fréquence est le paramètre le plus sensible à l'augmentation de la vitesse. L'accéléromètre peut être un appareil utile pour effectuer des comparaisons, même s'il a été constaté qu'audelà d'une certaine vitesse, l'augmentation se fera principalement par l'augmentation de l'amplitude et non de la fréquence. 
Ainsi, cette étude, réalisée sur tapis roulant montre que la fréquence d'appui est fortement corrélée à la vitesse pour un individu donné $\left(\mathrm{r}^{2}\right.$ compris entre 0.90 et 1 selon les individus), mais que la méthode des à-coups selon un seul axe n'est pas fiable, à cause d'une trop grande variabilité interindividuelle dans l'analyse du signal. En effet, si chez un même individu, la fréquence augmente avec la vitesse, on peut par contre observer des fréquences très différentes chez deux individus qui courent à la même vitesse. Dans une seconde étude sur tapis, en plaçant un accéléromètre tri-axe sur les lombaires (L4-L5), Wixted et al. (2007) trouvent une forte corrélation individuelle entre la vitesse de course et le nombre d'à-coups $\left(\mathrm{R}^{2}=0,96\right)$.

Dans une autre étude (Neville et al., 2011) sur tapis, les coureurs portent une veste spéciale dans laquelle une centrale, comprenant un GPS et un accéléromètre tri-axial à $100 \mathrm{~Hz}$, a été glissée vers le milieu du dos. Ils doivent réaliser des séries de $50 \mathrm{~m}$ à vitesse constante en démarrant à $8 \mathrm{~km} / \mathrm{h}$ pour finir à $30 \mathrm{~km} / \mathrm{h}$. La fréquence d'appui augmente de 2,9 à 3,9 Hz entre $15 \mathrm{~km} /$ et $30 \mathrm{~km} / \mathrm{h}$. Selon les auteurs, la corrélation entre ces deux variables est suffisante $(\mathrm{r}=0,896)$ pour estimer la vitesse. Toutefois, notons que le fait que 2 variables soient corrélées ne présage en rien que ces dernières soient validées. D'autant plus que la qualité de mesure de la vitesse par GPS peut aussi varier selon les conditions de mesure (météo, virage. etc.).

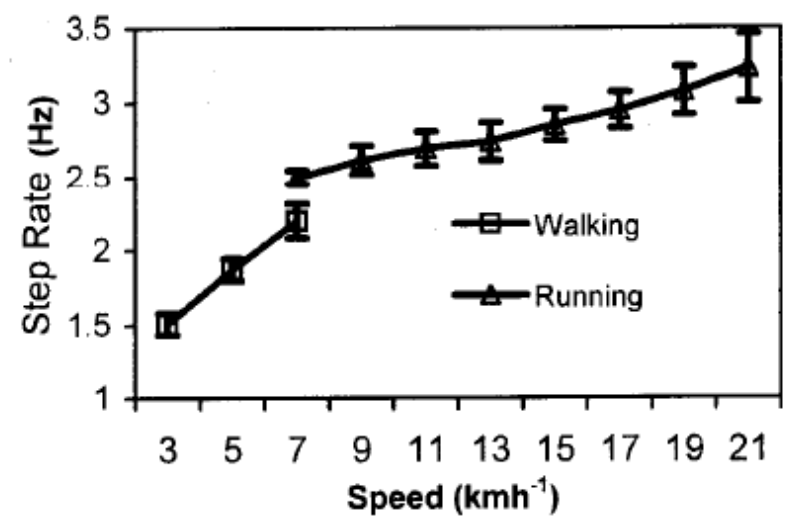

Figure 8. Augmentation de la fréquence d'appui en fonction de la vitesse. (D’après Wixted et al., 2005)

\subsection{Les paramètres de la foulée}

\subsubsection{La fréquence d'appui}

Une dizaine d'études ont tenté de mesurer la fréquence d'appui pendant la course. Les méthodes sont basées soit sur une transformation de Fourier des données 
accéléromètriques, soit en identifiant l'accélération verticale associée à l'impact du pied au sol. Pour ce faire, l'accéléromètre est attaché aux membres inférieurs. Neville utilise la méthode dite du croisement du zéro qui consiste à identifier dans le plan antéropostérieur chaque croisement avec la valeur zéro comme étant un impact au sol. Il est ensuite divisé par le temps pour en déduire la fréquence d'appui (Neville et al., 2011). Cette méthode montre des résultats fiables lorsque l'on compare avec la vitesse mesurée par chronomètre. La fréquence augmente de manière linéaire (Neville et al., 2011) avec la vitesse $\left(\mathrm{r}^{2}=0,96\right)$ et avec différentes vitesses mesurées par GPS $\left(\mathrm{r}^{2}=0,84\right)$. Il faut cependant noter que la méthode de mesure de la vitesse par chronomètre reste subjective et ne semble fonctionner que si un expert de cette tâche réalise la mesure (Vicente-Rodríguez et al., 2011).

Une autre solution est proposée par Bichler (Bichler et al., 2012) en utilisant un couplage accéléromètre et gyroscope pour identifier la fréquence d'appui. Ils cherchent à identifier la rotation du pied avant, pendant et après l'appui afin d'en déduire certains paramètres de la foulée comme la fréquence. Ainsi, l'appui est identifié comme la rotation en dessous du seuil de $1 \mathrm{rad} / \mathrm{s}$ couplé avec la donnée accéléromètrique du pic à l'impact au sol. Selon la méthode utilisée, l'appui est considéré soit comme le temps de contact au sol (accéléromètre) soit comme le niveau de rotation (gyroscope). Ces deux approches montrent une très bonne fiabilité, quelque soient la vitesse, la fatigue ou les athlètes.

\subsubsection{Les paramètres temporels de la foulée}

Ces paramètres sont très précieux dans l'analyse de la foulée, comme le temps d'envol, le temps de contact, le temps de la foulée. L'objectif est de trouver les indicateurs clés qui détermineront le début du contact du pied au sol et le moment du décollage. En utilisant une centrale inertielle, Stohrman et al. (2011) identifient le contact pied-sol comme étant le moment où l'accélération est inférieure à $2 g$. Une autre méthode consiste à analyser la composante d'accélération antéro-postérieure et à identifier les pics positifs importants comme étant le choc au sol (début du contact) et les pics plus petits comme étant le décollage. La correspondance entre une méthode ou l'accéléromètre est placé dans le dos et une capture du mouvement en 3D semble acceptable $(r=0,90)$ pour l'ensemble des paramètres temporels et ce quelle que soit la vitesse. Des résultats similaires (Purcell et al., 2005) sont trouvés en plaçant l'accéléromètre sur le tibia et en comparant les valeurs avec celles d'une plateforme de force $(r=0,89)$. Encore une fois, précisons qu'une 'une corrélation entre 2 mesures ne préfigure pas de la validité des résultats.

Dans une étude avec des sprinteurs amateurs et élites (Bergamini et al., 2012), il a été montré que l'utilisation d'une centrale inertielle, incluant un accéléromètre 3D et un gyroscope 3D (FreeSense) placée dans le bas du dos (vertèbre L1) permet une estimation de la durée de l'appui et de l'envol. La mesure est réalisée sur l'amplitude du signal de la vitesse angulaire et de sa dérivée seconde. Le pic de vitesse angulaire est consistant quelques soient les athlètes, les essais ou l'expertise. En effet ce pic est associé à une rotation du tronc dans l'axe longitudinal. Il permet 
d'estimer la durée de la foulée (avec une précision $<2 \%$ pour l'envol et $<8 \%$ pour l'appui) mais pas le moment de la pose du pied ou du décollage.

\subsubsection{La longueur de la foulée}

Cette variable, importante est étudiée notamment comme un marqueur de la fatigue ou comme variable liée à la raideur des membres inférieurs. La méthode utilisée dans deux études (Mercer et al., 2002 ; 2003) consiste à diviser la vitesse du tapis roulant par la fréquence, selon la méthode de mesure précédemment expliquée. Une autre étude (Bichler et al., 2012) montre une différence de seulement $0,01 \mathrm{~cm}$ dans la mesure de la longueur de la foulée comparativement à une analyse cinématique $2 \mathrm{D}$, mais sans préciser la méthode utilisée pour ce faire. Les rares études notamment sur piste laissent un champ encore peu exploité de la mesure de cette variable par accélérométrie en situation écologique.

\section{Bibliographie}

Atkinson G., Nevill A.M. (1998). Measurement Error ( Reliability ). Variables Relevant to Sports Medicine, vol. 26, $\mathrm{n}^{\circ}$ 4, p. 217-238.

Bampouras T. M., Relph N. S., Orme D., Esformes J. I. (2013). Validity and reliability of the Myotest Pro wireless accelerometer in squat jumps. Isokinetics and Exercise Science, vol. $21, \mathrm{n}^{\circ} 2, \mathrm{p} .101-105$.

Bergamini E., Picerno P., Pillet H., Natta F., Thoreux P., Camomilla V. (2012). Estimation of temporal parameters during sprint running using a trunk-mounted inertial measurement unit. Journal of biomechanics, vol. 45, n 6, p. 1123-1126.

Bichler S., Ogris G., Kremser V., Schwab F., Knott S., Baca A. (2012). Towards highprecision IMU/GPS-based stride-parameter determination in an outdoor runners' scenario. Procedia Engineering, vol. 34, p. 592-597.

Bland J.M. et Altman D.G. (1990). A note on the use of the intraclass correlation coefficient in the evaluation of agreement between two methods of measurement. Computers in biology and medicine, vol. 20, $\mathrm{n}^{\circ} 5, \mathrm{p} .337-340$.

Boji I., Boris Đ., Dimi A., Bubanj S., Stanković R., Bubanj R., Dimić A. (2010). Reliability of myotest tested by a countermovement jump. Acta Kinesiologica, vol. 4, n 2, p. 46-48.

Casartelli N., Müller R., Maffiuletti N.A. (2010). Validity and reliability of the myotest accelerometric system for the assessment of vertical jump height. The Journal of Strength \& Conditioning Research, vol. 24, $\mathrm{n}^{\circ} 11, \mathrm{p} .3186-3193$.

Castagna C., Ganzetti M., Ditroilo M., Giovannelli M., Rocchetti A., Manzi V. (2013). Concurrent Validity of Vertical Jump Performance Assessment Systems. The Journal of Strength \& Conditioning Research, vol. 27, n 3, p. 761-768. 
Choukou M.A., Laffaye G., Taiar R. (2014). Reliability and Validity of an accelerometric system for assessing vertical jumping performance. Biology of Sport, vol. 31, $\mathrm{n}^{\circ}$ 1, p. 55 62 .

Crewther B. T., Kilduff L. P., Cunningham D., Cook C., Yang G.-Z. (2010). Validity of two kinematic systems for calculating force and power during squat jumps. British Journal of Sports Medicine, vol. 44, n 14, p.i26-i26.

Fox E.L., Mathews D.K. (1974). Interval training: conditioning for sports and general fitness, Saunders Philadelphia.

Glatthorn J.F. et al. (2011). Validity and reliability of Optojump photoelectric cells for estimating vertical jump height. The Journal of Strength \& Conditioning Research, vol. $25, n^{\circ} 2$, p. 556-560.

Haff G. G., Ruben R., Saffel H., McCrory J. L., Cormie P., Sands W. A., Stone M. H. (2011). Reliability of Accelerometer Based Performance Measurements During Countermovement Vertical Jumps and the Influence of Sampling Frequency. The Journal of Strength \& Conditioning Research, vol. 25, p. S92.

Harman E. A., Rosenstein M. T., Frykman P. N., Rosenstein R. M., Kraemer W. J. (1991). Estimation of human power output from vertical jump. The Journal of Strength \& Conditioning Research, vol. 5, $\mathrm{n}^{\circ}$ 3, p. 116-120.

Van Ingen Schenau G.J., Bobbert M.F., De Haan A. (1997). Does elastic energy enhance work and efficiency in the stretch-shortening cycle? Journal of Applied Biomechanics, vol. 13 , p. 389-415.

Jidovtseff B., Apolloni J. et al. (2012a). Modelling relationships between jump height, ground contact time, reactivity and stiffness. In 8th International Conference on Strength Training. The Norwegian School of Sport Sciences, p. 183-184.

Jidovtseff B., Rodriguez de la Cruz C. et al. (2012b). influence of fatigue on the stride characteristics during an intense endurance runtest. In Abstract book of 17th ECSS Congress.

Johnson D.L. et Bahamonde R. (1996). Power output estimate in university athletes. The Journal of Strength \& Conditioning Research, vol. 10, $\mathrm{n}^{\circ} 3$, p.161-166.

Laffaye G., Bardy B., Taiar R. (2006). Upper-limb motion and drop jump: effect of expertise. Journal of Sports Medicine and Physical Fitness, vol. 46, n 2, p. 238-247.

McMaster D. T. W., Gill N. D., Cronin J. B., McGuigan M. R. (2013). Is wireless accelerometry a viable measurement system for assessing vertical jump performance? Sports Technology, vol. 6, $\mathrm{n}^{\circ} 2$, p. 86-96.

Mercer J. A., Bates B. T., Dufek J. S., Hreljac A. (2003). Characteristics of shock attenuation during fatigued running. Journal of Sports Science, vol. 21, n 11, p. 911-919. 
Mercer J. A., Vance J., Hreljac A., Hamill J. (2002). Relationship between shock attenuation and stride length during running at different velocities. European Journal of Applied Physiology, vol. 87, $\mathrm{n}^{\circ} 4-5$, p. 403-408.

Neville J., Rowlands D., Wixted A., James D. (2011). Determining over ground running speed using inertial sensors. Procedia Engineering, vol. 13, p.487-492.

Nuzzo J.L., Anning J.H., Scharfenberg J.M. (2011). The reliability of three devices used for measuring vertical jump height. Journal of Strength and Conditioning Research / National Strength \& Conditioning Association, vol. 25, n 9, p. 2580-2590.

Purcell B., Channells J., James D., Barrett R. (2005). Use of accelerometers for detecting foot-ground contact time during running. In Microelectronics, MEMS, and Nanotechnology. International Society for Optics and Photonics, p. 603-615.

Samozino P., Morin J.-B., Hintzy F., Belli A. (2008). A simple method for measuring force, velocity and power output during squat jump. Journal of biomechanics, vol. $41, \mathrm{n}^{\circ} 14$, p. $294-2945$.

Sargent D.A. (1921). The physical test of a man. American Physical Education Review, vol. 26, n 4 , p. 188-194.

Sayers S. P., Harackiewicz D. V, Harman E. A., Frykman P. N., Rosenstein M. T. (1999). Cross-validation of three jump power equations. Medicine and Science in Sports and Exercise, vol. $31, \mathrm{n}^{\circ}$ 4, p. 572-577.

Strohrmann C., Harms H., Troster G. (2011). What do sensors know about your running performance? In Wearable Computers (ISWC), 2011 15th Annual International Symposium on. IEEE, p. 101-104.

Vicente-Rodríguez G., Rey-López J. P., Ruíz J. R., Jiménez-Pavón D., Bergman P., Ciarapica D., Moreno L. A. (2011). Interrater Reliability and Time Measurement Validity of SpeedAgility Field Tests in Adolescents. The Journal of Strength \& Conditioning Research, vol. $25, n^{\circ} 7$, p. $2059-2063$.

Wixted A. J., Thiel D. V, Hahn A. G., Gore C. J., Pyne D. B., James D. A. (2007). Measurement of energy expenditure in elite athletes using MEMS-based triaxial accelerometers. Sensors Journal, IEEE, vol. 7, n 4, p. 481-488.

Wixted A., Thiel D., James D., Hahn A., Gore C., Pyne D. (2005). Signal processing for estimating energy expenditure of elite athletes using triaxial accelerometers. Sensors, 2005 IEEE. IEEE. 